The Bangladesh Veterinarian (2010) 27(1) : 1 - 7

\title{
Newcastle disease vaccination regimen comprising both Lentogenic and Mesogenic strains is more effective than Lentogenic strain only
}

\author{
L. R. Barman ${ }^{1 *}$, M. N. Islam, M. F. Flensburg'2, A. Permin², S. L. Petersen² and \\ M. R. Islam \\ Department of Pathology, Faculty of Veterinary Science, Bangladesh Agricultural \\ University, Mymensingh-2202, Bangladesh
}

\begin{abstract}
Two vaccination regimens against Newcastle disease were compared. A total of 180 crossbred (RIR $\times$ Fayoumi) day-old chicks were obtained from the government Poultry Farm. At three days of age, 10 birds were randomly selected and killed to obtain blood samples for serum collection. The remaining 170 birds were divided into three groups. Group A, (55) received a locally produced live lentogenic Newcastle disease virus (NDV) vaccine called "Baby Chick Ranikhet Disease Vaccine" (BCRDV) intraocularly at three and 28 days of age followed by a live mesogenic NDV vaccine namely "Ranikhet Disease Vaccine" (RDV) intramuscularly at 60 days of age. Group B, (55) was vaccinated with an imported live lentogenic commercial vaccine intraocularly at three, 28 and 60 days of age. Group C, (60) served as unvaccinated control. Further blood samples were collected on day 28, 60 and 74 from all groups. All the sera were tested for haemagglutination inhibition (HI) antibody titre to NDV. At 75 days of age, 30 birds from each of the two vaccinated groups and 32 birds from the unvaccinated group were challenged with a velogenic viscerotropic field isolate of NDV. The chicks had a high level of maternal HI antibodies at three days of age with a geometric mean titre (GMT) of $\log _{2} 4.8$, but that gradually declined in the unvaccinated group. Following vaccination there was no remarkable rise in the antibody titre at 28 and 60 days of age (GMT> $\left.\log _{2} 3.5\right)$, but at 74 days of age, the GMT of HI antibody in Group A $\left(\log _{2} 5.9\right)$ was significantly higher than that in the Group B $\left(\log _{2} 4.1\right)$. Following challenge, $100 \%$ morbidity and $65.6 \%$ mortality were observed in the unvaccinated group. Among the vaccinated groups, only $16.7 \%$ morbidity and no mortality were noticed in Group A but $43.3 \%$ morbidity and $10 \%$ mortality were observed in Group B. It is suggested that a vaccination programme based on lentogenic priming and mesogenic booster gave better protection against velogenic viscerotropic NDV challenge than that based on lentogenic vaccine only. (Bangl. vet. 2010. Vol. 27, No. 1, 1 - 7)
\end{abstract}

\section{Introduction}

Newcastle disease (ND) or Ranikhet disease is an economically important disease of chickens in most parts of the world. Newcastle disease virus (NDV) belongs to the genus Avulavirus in the family Paramyxoviridae. Based on the

\footnotetext{
${ }^{1}$ Mirpur Central Poultry Farm, Department of Livestock Services, Dhaka, Bangladesh

2 The Royal Veterinary and Agricultural University, DK-1870 Frederiksberg C., Denmark

* Correspondence E-mail :- lalitalalita2002@hotmail.com
} 
pathogenicity, NDVs are categorized into the most virulent velogenic, moderately virulent mesogenic, less virulent lentogenic or avirulent pathotypes (Alexander, 1997). Velogenic viscerotropic NDV is endemic in Bangladesh and causes mortality in both village chickens (Barman et al., 2010) and commercial poultry farms (Islam et al., 1998; Talha et al., 2001). Vaccination is the most effective means of protecting chickens against ND and is routinely practised in most parts of the world. The choice of vaccine types as well as the programme of vaccination is influenced by a variety of factors, such as immunogenicity of vaccine virus, virulence of field virus, routes of vaccine administration and the age of birds to be vaccinated. Both live and inactivated NDV vaccines are available. Avirulent or mild lentogenic strains of NDV are most commonly used as vaccines. However, in some parts of the world like South Asia where velogenic strains are endemic, moderately virulent mesogenic strains are also used as vaccines.

In Bangladesh, the Department of Livestock Services (DLS) produces NDV vaccines and distributes the vaccines at a subsidised cost. The vaccination programme suggested by DLS against ND includes intra-ocular administration of live lentogenic virus vaccine (F strain), called "Baby Chick Ranikhet Disease Vaccine" (BCRDV), in young chicks, while grower and adult birds are provided with live mesogenic virus (M strain) vaccine, called "Ranikhet Disease Vaccine" (RDV), by intramuscular injection. Some commercial poultry farmers of Bangladesh also use various imported live vaccines containing lentogenic La Sota or B1 strains, and depend solely on lentogenic vaccines. The aim of the present study was to compare the efficacy of two vaccination regimens based on either a commercial lentogenic vaccine alone or a combination of both lentogenic BCRDV and mesogenic RDV vaccines produced by DLS.

\section{Materials and Methods}

\section{Chicks and eggs}

Day-old crossbred (RIR $\times$ Fayoumi) chicks, locally known as Sonali chicks, were collected from the Government Poultry Farm, Mirpur, Dhaka. Chicks came from a parent stock that had been vaccinated against ND. Eggs for virus isolation and titration were collected from the Poultry Farm at Bangladesh Agricultural University.

\section{NDV Vaccines}

The following ND vaccines were used in the experiment :

(a) BCRDV : Freeze-dried culture of lentogenic F strain produced by the DLS, Bangladesh.

(b) RDV : Freeze-dried culture of mesogenic $M$ strain, produced by DLS, Bangladesh.

(c) Commercial vaccine : An imported vaccine containing freeze-dried culture of lentogenic La Sota strain. 
NDV isolate for challenge infection

A velogenic viscerotropic NDV field strain was isolated from a village chicken during an outbreak in Mymensingh District of Bangladesh (Barman et al., 2010). The virus was passaged twice in embryonated chicken eggs by allantoic route of inoculation. The pooled allantoic fluid obtained from the last passage had a titre of $\log _{10} 10 \mathrm{ELD}_{50}$ per $\mathrm{ml}$ and had the mean embryo death time of 56 hours when titrated in 10-day-old embryonated chicken eggs by standard method (Anon, 1996).

\section{Experimental design}

The experiment was conducted at the Department of Pathology of Bangladesh Agricultural University, Mymensingh. A total of 180 chicks were raised from day-old in relative isolation and supplied with feed and clean water ad libitum. At three days of age, 10 birds were randomly selected and killed to obtain blood samples for serum collection. The remaining 170 birds were divided into three groups. One group (Group A, 55 birds) was vaccinated intra-ocularly with BCRDV at day three and day 28 and intramuscularly with RDV at day 60; another group (Group B, 55 birds) was vaccinated with the commercial vaccine at day three, day 28 and day 60; and the third group (Group C, 60 birds) was kept as unvaccinated control. Birds of all three groups also received an infectious bursal disease virus vaccine at day 14 and day 21 by intra-ocular route. Blood samples were collected for serum from 10 birds randomly selected from each group at day 28 and day 60 (days of booster vaccination) and day 74 (the day before challenge). At 75 days of age, 30 birds each from the two vaccinated groups and 32 birds from the unvaccinated group were randomly selected, transferred to a new shed and challenged with a velogenic viscerotropic local NDV isolate by intramuscular injection. Each bird received $1 \mathrm{ml}$ virus suspension containing $\log _{10} 5.0 \mathrm{EID}_{50}$. The birds were closely observed for clinical signs and mortality up to 90 days of age (15 days after challenge). Detailed necropsy was conducted on dead birds.

Serology

Serum samples obtained at day three, 28, 60 and 74 were tested for NDV antibodies by haemagglutination inhibition $(\mathrm{HI})$ test following the standard procedure (Anon, 1996). All sera were tested in two-fold serial dilutions.

\section{Statistical analysis}

$\log _{2}$ geometric mean titre (GMT) of HI antibodies was calculated for each occasion in each group. End-point HI titres of different groups at 28, 60 and 74 days of age were compared by $t$-test. The morbidity and mortality of different groups following challenge infection were compared by $x^{2}$ analysis.

\section{Results and Discussion}

Antibody response

The GMT of HI antibodies in three groups at different times are presented in Fig. 1. The chicks had a high level of maternal antibodies. The GMT of maternal HI 
antibodies at three days of age was $\log _{2} 4.8$. In the unvaccinated group (Group C) the GMT of maternal antibody dropped to $\log _{2} 0.66$ at 28 days of age and was almost undetectable at 60 and 74 days of age. The HI antibody titres in the vaccinated groups (Groups A and B) at 28 and 60 days of age were also not very high $\left(<\log _{2} 3.5\right)$ but were higher than in the unvaccinated group. At 74 days of age, the GMT of HI antibodies in Group A increased remarkably to $\log _{2} 5.9$ and in Group B to $\log _{2} 4.1$. The HI antibody level at 74 days of age was significantly higher $(\mathrm{P}<0.01)$ in Group A than in Group B.

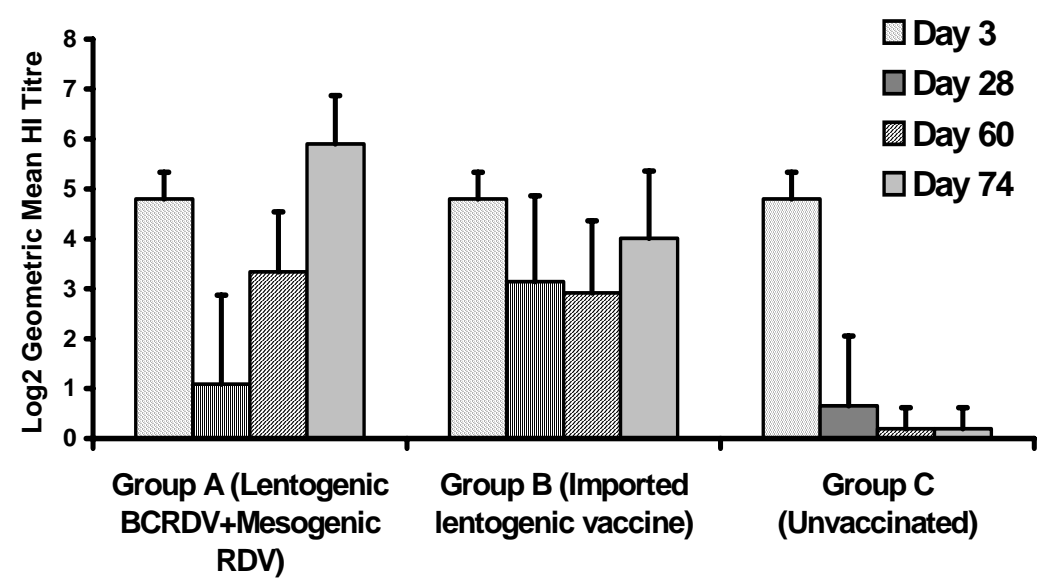

Fig. 1. Geometric mean HI titre in different groups of chickens following NDV vaccination. Vaccines were given at 3,28 and 60 days of age

\section{Morbidity and mortality after challenge}

Results of the challenge infection are shown in Fig. 2. In unvaccinated Group C, morbidity and mortality were $100 \%$ and $65.6 \%$, respectively. In Group A morbidity was $16.7 \%$, but no mortality was recorded. However, in Group B morbidity and mortality were $43.3 \%$ and $10.0 \%$, respectively. The morbidity was significantly $(\mathrm{P}<0.01)$ higher in the unvaccinated group than in the vaccinated groups. The morbidity was significantly $(\mathrm{P}<0.05)$ higher in Group B than in Group A. Similarly, the mortality was significantly $(\mathrm{P}<0.01)$ higher in the unvaccinated group than in the vaccinated groups.

\section{Clinical signs and post-mortem lesions}

Clinical signs were first noticed in the unvaccinated group as early as 48 hours after challenge. Marked depression, whitish diarrhoea, head tremor and twisting, occasional paralysis and lateral recumbency were the principal clinical signs. Mortality was first noticed on day three after challenge, and continued until day eight. 
Most of the dead birds showed severe lesions with haemorrhages in the gastrointestinal tract. About $90 \%$ of the dead birds had button-like ulcers in the caecal tonsils. More than $80 \%$ of the dead birds showed haemorrhagic lesions in the tips of the proventricular glands, and about $10 \%$ had congestion or haemorrhage in the lungs and trachea. Splenomegaly along with white necrotic spots was also common.

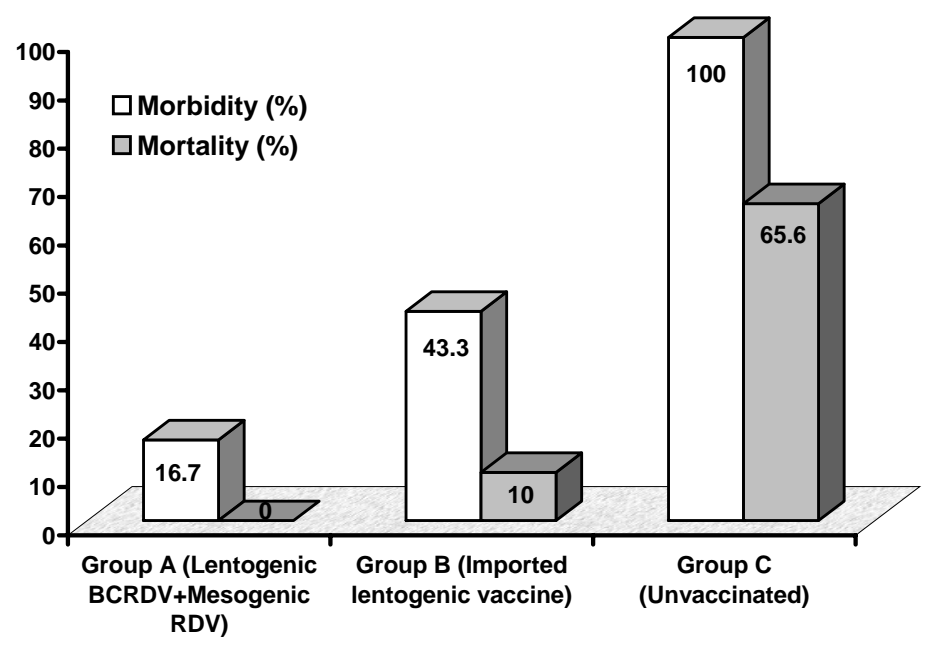

Fig. 2. Morbidity and mortality of chickens in vaccinated and unvaccinated groups following challenge with a velogenic viscerotropic NDV field isolate

Two different vaccination regimens, based on either a combination of lentogenic BCRDV ('F' strain) and mesogenic RDV (Mukteswer 'M' strain) vaccines of DLS or only an imported lentogenic (La Sota strain) vaccine, were compared. Three doses of vaccines were administered on three, 28 and 60 days of age, as commonly practised in Bangladesh. Crossbred 'Sonali' chicks were used in this experiment as they are considered as potential high-yielding village chickens in Bangladesh under scavenging or semi-intensive system.

The chicks had a high level of maternal antibodies, but the level of maternal antibodies gradually declined as observed in the unvaccinated control group. The HI antibody levels did not increase remarkably following administration of the first two doses of lentogenic BCRDV in Group A or the commercial lentogenic vaccine in Group B. Relatively low antibody response following the primary vaccination at three days of age could be due to neutralisation of the vaccine virus by high level of maternal antibodies. If this was the case, then the possibility of delaying the primary vaccination may be considered. Higher antibody level in Group B than in group A at 28 days of age could indicate that La Sota strain of the imported vaccine might be more invasive than F strain of BCRDV in presence of maternal antibodies. However, 
this hypothesis needs to be further tested. Westbury et al. (1984) and Rehmani (1996) suggested that La Sota strain provides good immunity in presence of maternal antibodies. However, the reason for low antibody response observed in the present study even after the booster vaccination given at 28 days of age could not be readily explained. Immunosuppression resulting from the two doses of live intermediate IBDV vaccines could be an explanation. IBDV vaccines were given at 14 and 21 days of age, as commonly practised in intensive poultry farms of Bangladesh. This hypothesis needs to be further investigated.

The use of RDV as the third dose of vaccine at 60 days of age in Group A resulted in significant increase of the HI antibody level at 74 days of age. However, the antibody level in Group B did not increase much after the third dose of the commercial vaccine and the $\mathrm{HI}$ antibody levels in the two vaccinated groups were also significantly different at 74 days of age. Sharp rise in the antibody level following the administration of RDV may be related to the virus strain used in this vaccine. RDV contains a more invasive mesogenic Mukteswer (M) strain.

Schmidt and Schmidt (1955) established a relationship between HI titre and the protection capacity of birds from a study conducted over a 10-month period following vaccination. They found that $8.5-95 \%$ of birds having titres up to $\log _{2} 4.0$ failed to resist the challenge infection against virulent NDV, whereas those having a titre $\log _{2} 5.0$ or above resisted the challenge infection, and thus they considered the $\mathrm{HI}$ titre $\log _{2} 5.0$ and above to be protective. All birds in Group A receiving two doses of lentogenic BCRDV and one dose of mesogenic RDV had protective level of antibodies (HI titre > $\log _{2} 5.0$ ) before challenge; whereas, only $40 \%$ of the birds in Group B that received three doses of lentogenic commercial vaccine had protective level of antibodies before challenge (raw data not shown) and the GMT was also below the protective level. This was partially reflected in the challenge experiment as significantly more birds $(43.3 \%)$ became clinically affected in Group B than in Group A (16.7\%). Furthermore, there was no mortality in Group A while 10\% mortality was observed in Group B.

In the challenge experiment, use of lentogenic BCRDV plus mesogenic RDV in combination (Group A) provided 100\% protection against mortality. Despite the low level of antibody, lentogenic commercial vaccine alone (Group B) also gave $90 \%$ protection against mortality. This would indicate that in addition to humoral immunity, cell-mediated immune effector mechanisms might be involved in the protection against NDV.

Velogenic viscerotropic NDV is endemic in Bangladesh. This necessitates rigorous vaccination programme. A schedule consisting of two doses of lentogenic vaccine (BCRDV) followed by mesogenic vaccine (RDV) given at regular intervals is a common practice in Bangladesh. The findings of the present study would justify this programme. The present study would suggest that vaccination programme based on lentogenic vaccine only may not be enough to fully protect birds from velogenic viscerotropic NDV challenge unless the birds are boosted with a mesogenic 
vaccine. It remains to be investigated whether an inactivated NDV vaccine could replace the live RDV.

\section{References}

Alexander DJ 1997: Newcastle disease and other avian paramyxoviridae infections. In: Calnek BW, Barnes HJ, Beared CW, McDougald LR Saif YM [Editors], Diseases of Poultry, $10^{\text {th }}$ Edn. Iowa State University Press, Iowa. pp. 541-569.

Anon 1996: Newcastle disease. In: Manual of Standards for Diagnostic Tests and Vaccines, $3^{\text {rd }}$ Edn. Office International des Epizooties, Paris. pp. 161-169.

Barman LR, Flensburg MF, Permin A, Madsen M, Petersen SL, Islam MR 2010: Studies on the epidemiology of Newcastle disease in village chickens in Bangladesh. [Manuscript submitted for publication].

Islam MR, Khan MAHNA, Das PM, Bari ASM 1998: Poultry diseases diagnosed at necropsy in 1997 and 1998 in the Department of Pathology, Bangladesh Agricultural University, Mymensingh. Programme and Abstracts of $5^{\text {th }}$ BSVER Annual Scientific Conference, Bangladesh Agricultural University, Mymensingh.

Rehmani SF 1996: Newcastle disease vaccination: a comparison of vaccines and routes of administration in Pakistan. Preventive Veterinary Medicine 25 241-248.

Schmidt U, Schmidt D 1955: Connection between haemagglutination-inhibition antibodies and immunity after vaccination against Newcastle disease. Archives of Experimental Veterinary Medicine 9 505-516.

Talha AFSM, Hossain MM, Chowdhury EH, Bari ASM, Islam MR, Das PM 2001: Poultry diseases occurring in Mymensingh district of Bangladesh. Bangladesh Veterinarian 18 20-23.

Westbury, HA, Parsons G, Allan WH 1984: Comparison of the immunogenicity of Newcastle disease virus strains V4, Hitchner B1 and La Sota in chickens. 2. Tests in chickens with maternal antibody to the virus. Australian Veterinary Journal 61 10-13. 